\title{
Genetic ablation of Bach1 gene enhances recovery from hyperoxic lung injury in newborn mice via transient upregulation of inflammatory genes
}

\author{
Masato Ito', Nobuhiko Nagano', Yukio Arai', Ryo Ogawa', Shingo Kobayashi', Yukiko Motojima', Hayato Go², \\ Masanori Tamura' ${ }^{1}$ Kazuhiko Igarashi ${ }^{3}$, Phyllis A. Dennery ${ }^{4}$ and Fumihiko Namba'
}

\begin{abstract}
BACKGROUND: BTB and CNC homology 1 (Bach1) is a transcriptional repressor of heme oxygenase $(\mathrm{HO})-1$. The effects of Bach1 disruption on hyperoxic lung injury in newborn mice have not been determined. We aimed to investigate the role of Bach1 in the newborns exposed to hyperoxia.

METHODS: Bach $1^{-1-}$ and WT newborn mice were exposed to $21 \%$ or $95 \%$ oxygen for $4 d$ and were then allowed to recover in room air. Lung histology was assessed and lung Bach1, HO-1, interleukin (IL)-6, and monocyte chemoattractant protein (MCP)-1 mRNA levels were evaluated using RT-PCR. Lung inflammatory cytokine levels were determined using cytometric bead arrays.
\end{abstract}

RESULTS: After 10 d recovery from neonatal hyperoxia, Bach $1^{-1-}$ mice showed improved lung alveolarization compared with WT. HO-1, IL-6, and MCP-1 mRNA levels and IL-6 and MCP-1 protein levels were significantly increased in the Bach $1^{-1-}$ lungs exposed to neonatal hyperoxia. Although an increase in apoptosis was observed in the Bach $1^{-1-}$ and WT lungs after neonatal hyperoxia, there were no differences in apoptosis between these groups.

CONCLUSION: Bach $1^{-1-}$ newborn mice were well-recovered from hyperoxia-induced lung injury. This effect is likely achieved by the antioxidant/anti-inflammatory activity of $\mathrm{HO}-1$ or by the transient overexpression of proinflammatory cytokines.

$B^{\text {pas }}$ PD is a chronic lung disease developed after oxygen inhalation therapy or mechanical ventilation usually occurring in certain premature infants or newborn infants with respiratory distress syndrome. Histologically, it is characterized by the unusual abnormalities of the bronchioles, such as metaplasia, decrease in alveolar number, and formation of cysts. The pathophysiology of this condition is complex and may involve a genetic predisposition, perinatal inflammation, or environmental toxin exposure. Frequently, the use of respiratory support, including supplemental oxygen and mechanical ventilation, is required in neonates with $\mathrm{BPD}(1,2)$. The animal model of BPD involves exposing newborn mice to hyperoxia.
In this model, researchers can specifically target the saccular phase, one of the immature stages of lung development, by exposing the newborn mice to hyperoxia because newborn mouse lungs are equivalent to the lung developmental stage of a human infant born at $26-28 \mathrm{wk}$ of gestation (3). Hyperoxia is one of the known risk factors for the development of BPD. In this mouse model, hyperoxic conditions can be easily reproduced and oxygen concentrations can be manipulated to study dose dependence. Therefore, continuous exposure of newborn rodents to high concentrations of oxygen has often been used to research the effects of hyperoxia on developing immature lungs (4). Hyperoxia causes significant changes to the morphology of lung tissue in newborn mice, including decreased septation of the alveoli, enlarged and simplified terminal air spaces, and a greater degree of pulmonary fibrosis (5-8).

Hyperoxia-induced lung injury is initiated by reactive oxygen species, and this is followed by the secretion of proinflammatory chemokines and cytokines by resident macrophages and epithelial cells. Neutrophils then migrate into the airspace (9), and macrophages (10) reduce inflammatory responses through the ingestion of apoptotic neutrophils and other dying cells or dead cell debris, thereby mitigating tissue damage (1113). The delayed removal of dying cells may directly affect the natural ability of the injured organism to shut down inflammation and initiate tissue repair (14).

$\mathrm{BTB}$ and CNC homology 1 (Bach1) is a transcriptional repressor of the heme oxygenase $(H O)-1$ and $\beta$-globin genes $(15,16)$. Heterodimers of the small Maf proteins and NF-E2-related factor 2 (Nrf2) activate HO-1 through binding to Maf-responsive elements. In contrast, heterodimers of the small Maf proteins and Bach1 repress Maf-responsive element-dependent transcription. The ability of Nrf2 to activate HO-1 expression is greatly reduced in the presence of Bach1 under normal conditions $(16,17)$. However, under various stress conditions, such as inflammation, oxidative stress, or the presence of heme and cadmium, Bach1 detaches from the Maf-responsive elements, allowing Nrf2 and small Maf proteins to bind. This results in the transcriptional activation of target genes, such as $\mathrm{HO}-1(18,19)$.

'Department of Pediatrics, Saitama Medical Center, Saitama Medical University, Saitama, Japan; ${ }^{2}$ Department of Pediatrics, Fukushima Medical University School of Medicine, Fukushima, Japan; ${ }^{3}$ Department of Biochemistry, Tohoku University Graduate School of Medicine, Sendai, Japan; ${ }^{4}$ Department of Pediatrics, The Warren Alpert Medical School of Brown University, Providence, Rhode Island. Correspondence: Fumihiko Namba (nambaf@saitama-med.ac.jp)

Received 25 April 2016; accepted 30 October 2016; advance online publication 22 March 2017. doi:10.1038/pr.2017.17 
In Bach1-null (Bach1 $\left.{ }^{--}\right)$mice, the transcription of HO-1 is constitutively upregulated, thus leading to increased HO-1 protein levels and enzymatic activity under normal conditions in several organs, including the heart, lungs, and liver (16). Previous studies have shown that $B a c h 1^{-1-}$ mice are protected from conditions that are caused by oxidative stress, including atherosclerosis and myocardial ischemia/reperfusion injury $(20,21)$. The underlining mechanism of the protection of Bach $1^{-1-}$ mice from oxidative stress can be explained, in part, by the upregulation of HO-1 activity (20); however, much remains to be elucidated. Bach $1^{-1-}$ adult mice have been shown to be well-protected against hyperoxic lung injury. This protection was demonstrated by studies showing that compared with WT mice, Bach $1^{-1-}$ mice survive significantly longer, have less tissue injury based on histological findings, less protein in their bronchoalveolar lavage fluid, and reduced levels of apoptosis (22). However, there are no previous reports investigating the effects of Bach1 deficiency on hyperoxia-induced lung injury in newborn mice.

Therefore, in this study, we tested the hypothesis that genetic ablation of the Bach1 gene ameliorates hyperoxia-induced lung injury in newborn mice via upregulation of its target genes.

\section{METHODS}

\section{Animals}

All procedures and protocols were approved by the Animal Care and Use Committee of Saitama Medical University (Permit no. 1502). Bach $1^{-/-}$mice, originally created by Sun et al. (16) and repeatedly backcrossed with C57BL/6 mice for at least 12 generations, were maintained in the animal facility at the Saitama Medical Center, Saitama Medical University. Heterozygous Bach1 knockout male and female mice were mated to produce $B a c h 1^{-/}$and WT littermates; genotyping was performed by PCR analysis of tail biopsies.

\section{Neonatal Hyperoxic Exposure and Recovery}

Neonatal pups were randomly assigned to normoxia (room air) or hyperoxia $\left(95 \% \mathrm{O}_{2}\right)$. Exposure to hyperoxia was conducted for $96 \mathrm{~h}$ in a chamber (BioSpherix, Redfield, NY) that allowed for the continuous monitoring and regulation of oxygen and carbon dioxide. Dams were switched every $24 \mathrm{~h}$ between normoxia and hyperoxia. The inside of the chamber was kept at atmospheric pressure, and mice were exposed to a $12 \mathrm{~h}$ light-dark cycle.

\section{Lung Tissue Collection}

Mice were anesthetized with an intraperitoneal injection of pentobarbital $(50 \mathrm{mg} / \mathrm{kg})$. After the pulmonary artery was perfused with phosphate buffered saline, the right lung was excised and snap-frozen with liquid nitrogen for RNA and protein analysis. The left lung was inflated through the trachea with $10 \%$ neutral-buffered formalin (Sigma-Aldrich, St. Louis, MO) at $25 \mathrm{~cm}$ gravity pressure and allowed to fix for $1 \mathrm{~min}$. The trachea was tied; the lung was then removed and fixed further overnight at $4^{\circ} \mathrm{C}$. Lung tissue was paraffin-embedded and 5-mm thick sections were mounted onto glass slides.

\section{Lung Histology and Morphometry}

To assess distal airspace maturation, computer-aided morphometric analysis was performed using ImageJ software version $1.49(\mathrm{NIH}$, Bethesda, MD) to measure the $\mathrm{Lm}$ and the number of secondary septa. Paraffin-embedded lung tissue sections were stained with hematoxylin and eosin. Lm, which is defined as the mean length of line segments on random test lines spanning the airspace between intersections of the line with the alveolar surface, was determined using light microscopy (23). Lm was assessed in six nonoverlapping fields of lung parenchyma in one tissue section per animal, and five animals per condition at each time point were examined. Lm was obtained by dividing the total length of a line drawn across the lung section by the total number of intercepts, until the number of intercepts reached 50 per each field. Elastin staining was performed using an elastic stain kit (Abcam, Cambridge, MA) according to the manufacturer's instructions. The number of secondary septa, where elastin was detected, was manually counted in six nonoverlapping fields of lung parenchyma in one tissue section per animal, and five animals per condition at each time point were examined. Lung tissue was also analyzed for terminal deoxynucleotidyl transferase nick-end labeling (TUNEL) using the in situ Apoptosis Detection Kit (Takara Bio, Shiga Japan) according to the manufacturer's protocol. The number of TUNEL-positive cells was manually counted in six nonoverlapping fields of lung parenchyma per animal, and five animals per condition at each time point were examined. Fields containing large vessels, conducting airways or sectioning artifacts were avoided for lung morphometry.

\section{RNA Extraction and quantitative RT-PCR Analysis}

Total RNA was extracted from five lung tissue samples per group as previously described (24). RNA (500 ng) was reverse-transcribed (High Capacity cDNA Reverse Transcription Kit, Applied Biosystems, Foster City, CA), and cDNA was used in each PCR reaction with primers for HO-1, interleukin (IL)-6, and monocyte chemoattractant protein (MCP)-1 (Applied Biosystems) and TaqMan Universal PCR Master Mix (Applied Biosystems). Analyses were performed using an Applied Biosystems 7,500 Fast Real-Time PCR System. The relative mRNA expression levels were determined using the comparative critical threshold method, and each sample was normalized to betaglucuronidase (Applied Biosystems).

\section{Cytokine Measurements in Lung Tissue}

Mouse IL-12, tumor necrosis factor (TNF)- $\alpha$, interferon (IFN)- $\gamma$, MCP-1, IL-10, and IL-6 levels were determined in lung tissue homogenates using the BD Cytometric Bead Array Mouse Inflammation Kit (BD Biosciences, San Diego, CA) according to the manufacturer's instructions. The samples were analyzed on a BD FACSVerse flow cytometer (BD Biosciences).

\section{Statistical Analysis}

All values were expressed as the mean \pm SEM or as box-and-whisker plots (the median, interquartile range, and range). Comparison between different groups was performed with SPSS software version 22.0 (SPSS, Chicago, IL) by one-way ANOVA followed by Tukey posthoc test for analyzing parametric data or Kruskal-Wallis test followed by Mann-Whitney $U$-test for analyzing nonparametric data. $P$-values $<0.05$ were considered to be significant.

\section{RESULTS}

\section{Alveolar Development is Impaired after Neonatal Hyperoxia in both WT and Bach $1^{-/-}$Mice at $4 \mathrm{~d}$ of Age}

In normal room air, mice develop well-organized terminal airways. In contrast, exposure of newborn mice to hyperoxia for $96 \mathrm{~h}$ impaired alveolar development, resulting in alveolar expansion and simplification (Figure 1a), and inhibited secondary septation (Figure 1b). The mean linear intercept (Lm) in both WT and Bach1 $1^{-/-}$mouse lungs exposed to neonatal hyperoxia was significantly longer than that in normoxic control lungs at $4 \mathrm{~d}$ of age (Figure 1c). The number of secondary septa in the lungs was also significantly decreased after neonatal hyperoxia in both WT and Bach1 $1^{-/-}$mice (Figure 1d). No significant differences in the Lm or the number of secondary septa were observed between 4-d-old WT and Bach $1^{-1-}$ mice exposed to either normoxia or hyperoxia.

\section{Impaired Alveolar Development in Bach $1^{-/-}$Mice Recovers at 14 d of Age}

When neonatal WT mice were exposed to hyperoxia for $4 \mathrm{~d}$ and then allowed to recover in normal room air for $10 \mathrm{~d}$, they 
a

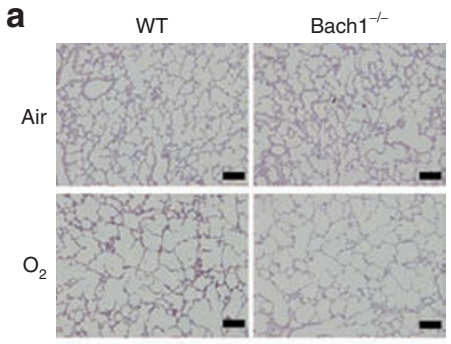

b

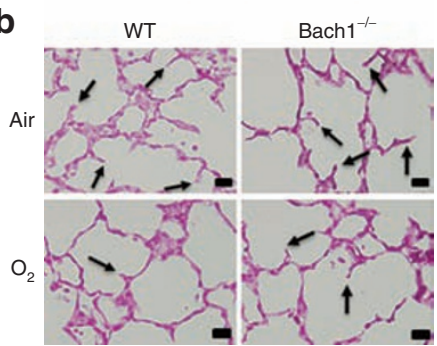

C

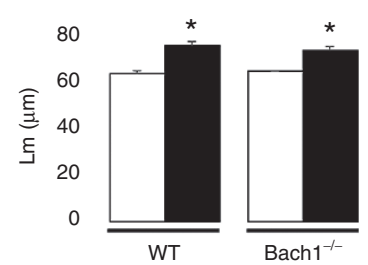

d

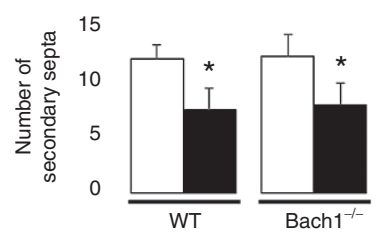

Figure 1. Alveolar development after neonatal hyperoxic exposure at $4 \mathrm{~d}$ of age. Exposure of newborn mice to hyperoxia for $96 \mathrm{~h}$ impaired alveolar development, resulting in alveolar expansion and simplification a) and inhibited secondary septation b. (a) hematoxylin and eosin (H\&E) stained histological sections from mice on days 4, Scale bar $=100 \mu \mathrm{m}$ (magnification: $\times 100)$. (b) Elastin stained histological sections from mice on days 4, Scale bar $=25 \mu \mathrm{m}$ (magnification: $\times 400$ ). Air: normoxia; $\mathrm{O}_{2}$ : hyperoxia. (c) Lm on days 4 ( $n=5$ animals in each group). Lm was assessed in six nonoverlapping fields of lung parenchyma in one tissue section per animal. Animals were exposed to air (open bars) or $\mathrm{O}_{2}$ (filled bars). Lm in both WT and $\mathrm{BaCh}^{-/-}$mouse lungs exposed to neonatal hyperoxia was significantly longer than that in normoxic controls at $4 \mathrm{~d}$ of age. (d) Number of secondary septa on days 4 ( $n=5$ animals in each group). The number of secondary septa was assessed in six nonoverlapping fields of lung parenchyma in one tissue section per animal. Animals were exposed to air (open bars) or $\mathrm{O}_{2}$ (filled bars). The number of secondary septa in the lungs was significantly decreased after neonatal hyperoxia in both WT and Bach $1^{-/-}$mice. Data are the mean \pm SEM. Lm: mean linear intercept. Comparison between different groups was performed by one-way ANOVA followed by Tukey test. ${ }^{*} P<0.05$ vs. Air.

still had a significantly longer Lm and less secondary septa compared with their normoxic counterparts at $14 \mathrm{~d}$ of age. In contrast, despite impaired alveolarization after neonatal hyperoxia at $4 \mathrm{~d}$ of age, $\mathrm{Bach}^{-/-}$mice had a significantly improved $\mathrm{Lm}$ and an increased number of secondary septa after room air recovery at $14 \mathrm{~d}$ of age compared with similarly exposed WT mice (Figure 2).

Hyperoxia-induced Apoptosis is Observed in the Lungs of both Bach $1^{-/-}$and WT Mice at $4 \mathrm{~d}$ of Age, but not at $14 \mathrm{~d}$ of Age To assess the antiapoptotic properties of the lungs of Bach1 $1^{-/-}$ mice, TUNEL assay was performed on lung sections of 4- and 14-d-old WT and Bach1 $1^{--}$mice. The number of TUNELpositive cells was significantly increased in the lungs of WT and $B a c h 1^{-/-}$mice after hyperoxic exposure compared with their normoxic counterparts at $4 \mathrm{~d}$ of age (Figure 3a,b). However, no significant differences in the number of TUNELpositive cells were observed between 4 -d-old WT and Bach1 $1^{-/}$ mice exposed to either normoxia or hyperoxia. At $14 \mathrm{~d}$ of age, there were no significant differences in the number of apoptotic cells in the lungs of WT and Bach $1^{-/-}$mice exposed to

a

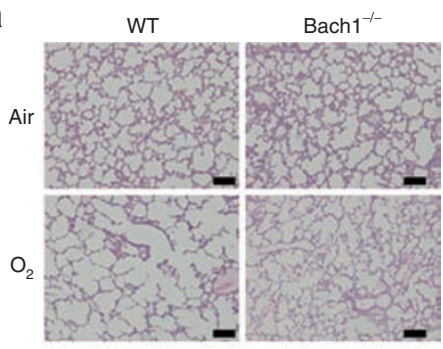

b

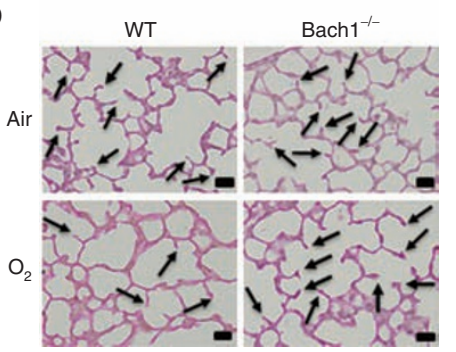

C

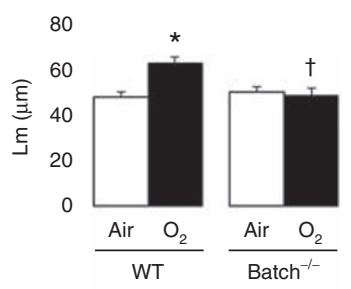

d

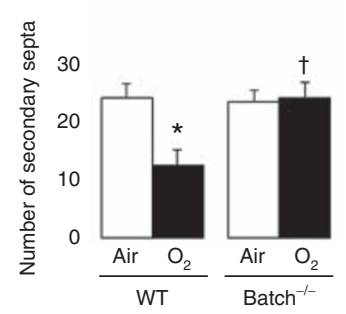

Figure 2. Alveolar development during recovery from neonatal hyperoxic exposure at $14 \mathrm{~d}$ of age. Impaired alveolar development in Bach $1^{-1-}$ mice recovers at $14 \mathrm{~d}$ of age. Bach $1^{--}$mice had a significantly improved $\mathrm{Lm}$ and an increased number of secondary septa after recovery from hyperoxia compared with WT. (a) Hematoxylin and eosin (H\&E) stained histological sections from mice on days 14, Scale bar $=100 \mu \mathrm{m}$ (magnification: $\times 100$ ). (b) Elastin stained histological sections from mice on days 14, Scale bar $=$ $25 \mu \mathrm{m}$ (magnification: $\times 400$ ). Air: normoxia; $\mathrm{O}_{2}$ : hyperoxia. (c) Lm on days 14 ( $n=5$ animals in each group). Lm was assessed in six nonoverlapping fields of lung parenchyma in one tissue section per animal. Animals were exposed to air (open bars) or $\mathrm{O}_{2}$ (filled bars). (d) Number of secondary septa on days 14 ( $n=5$ animals in each group). The number of secondary septa was assessed in six nonoverlapping fields of lung parenchyma in one tissue section per animal. Animals were exposed to air (open bars) or $\mathrm{O}_{2}$ (filled bars). Data are the mean \pm SEM. Comparison between different groups was performed by one-way ANOVA followed by Tukey test. ${ }^{*} P<$ 0.05 vs. Air, $+P<0.05$ vs. WT $\mathrm{O}_{2}$.

neonatal hyperoxia compared with the normoxic control mice (Figure 3a,c).

\section{Lung mRNA Expression Levels of HO-1 and IL-6 in Bach1 1-/- Mice Exposed to Neonatal Hyperoxia are Enhanced Compared with WT Mice}

Because Bach1 is reported to be a transcriptional repressor of the HO- 1 and $I L-6$ genes (22), lung mRNA expression levels of HO- 1 and IL- 6 were determined using q RT-PCR. Lung HO-1 and IL-6 mRNA expression levels were significantly increased in WT and Bach1 ${ }^{-/}$mice after a 4-d hyperoxic exposure, compared with normoxic controls. Bach $1^{-1-}$ mice exposed to neonatal hyperoxia also had significantly enhanced mRNA expression levels of HO- 1 and IL- 6 at $4 \mathrm{~d}$ of age compared with WT mice. After a 10-d recovery in room air (at $14 \mathrm{~d}$ of age), HO-1 and IL-6 mRNA expression levels were decreased to basal levels in WT and Bach $1^{-1-}$ mice (Figure 4).

\section{Effects of Bach1 Disruption on Inflammatory Cytokine Production in the Lungs after Exposure to Neonatal Hyperoxia} To evaluate the inflammatory response in the lungs during hyperoxic exposure, the inflammatory cytokine levels in 4-dold WT and $\mathrm{Bach}^{-/-}$mouse lungs exposed to either normoxia 
a

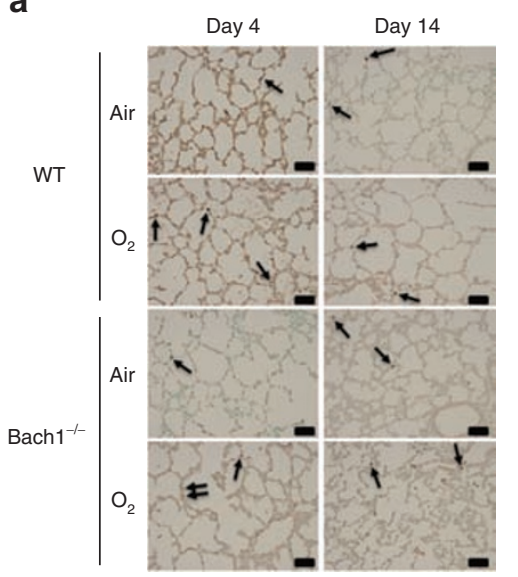

b

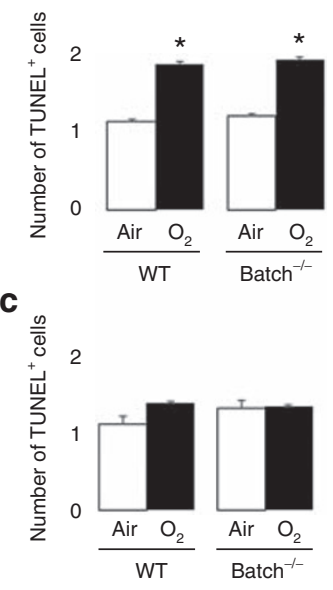

Figure 3. TUNEL staining of the lungs after neonatal hyperoxic exposure. Hyperoxia-induced apoptosis is observed in the lungs of both Bach1 $1^{-/-}$ and WT mice at $4 \mathrm{~d}$ of age, but not at $14 \mathrm{~d}$ of age. (a) TUNEL stained histological sections from animals, Scale bar $=50 \mu \mathrm{m}$ (magnification: $\times 200$ ). Air: normoxia; $\mathrm{O}_{2}$ : hyperoxia. (b) Number of TUNEL positive cells on days 4 ( $n=$ 5 animals in each group). The number of TUNEL positive cells was assessed in six nonoverlapping fields of lung parenchyma in one tissue section per animal. Animals were exposed to air (open bars) or $\mathrm{O}_{2}$ (filled bars). (c) Number of TUNEL positive cells on days 14 ( $n=5$ animals in each group). The number of TUNEL positive cells was assessed in six nonoverlapping fields of lung parenchyma in one tissue section per animal. Animals were exposed to air (open bars) or $\mathrm{O}_{2}$ (filled bars). Data are the mean $\pm \mathrm{SEM}$. Comparison between different groups was performed by one-way ANOVA followed by Tukey test. ${ }^{*} P<0.05$ vs. Air. TUNEL, terminal deoxynucleotidyl transferase nick-end labeling.

or hyperoxia for $4 \mathrm{~d}$ were measured using the Cytometric Bead Array Mouse Inflammation Kit. Furthermore, time course of changes in cytokine protein levels in the lungs after exposure to neonatal hyperoxia was examined. No significant differences were observed in the protein levels of TNF- $\alpha$, IFN- $\gamma$, IL-10, or IL-12 in WT and Bach1 ${ }^{-1-}$ mouse lungs after either normoxic or hyperoxic exposure. However, both IL-6 and MCP-1 levels in the $\mathrm{Bach}^{-/-}$mice lung were significantly higher than those of the WT mice lung following a 4-d hyperoxic exposure (Figure 5). This increase in protein expression levels of IL- 6 and MCP-1 was transient and these cytokine levels were decreased to the basal levels $24 \mathrm{~h}$ after exposure to hyperoxia (see Supplementary Figure S1 online).

\section{Lung mRNA Expression Levels of MCP-1 in Bach $1^{-/-}$Mice Exposed to Neonatal Hyperoxia are Enhanced Compared with WT Mice}

Because MCP-1 levels in the Bach $1^{-/}$mouse lungs were significantly higher than those in the lungs of WT mice, we also measured the mRNA expression levels of MCP-1 in the lungs using qRT-PCR. The MCP-1 mRNA expression level in the lungs of $\mathrm{WT}$ and $B a c h 1^{-/-}$mice was significantly increased after $4 \mathrm{~d}$ of hyperoxic exposure compared with normoxic exposure. Furthermore, Bach1 $1^{-/-}$mice exposed to neonatal hyperoxia had a significantly elevated mRNA expression level of MCP-1 at $4 \mathrm{~d}$ of age compared with WT mice. After $10 \mathrm{~d}$ of recovery in room air (at $14 \mathrm{~d}$ of age), the MCP-1 mRNA

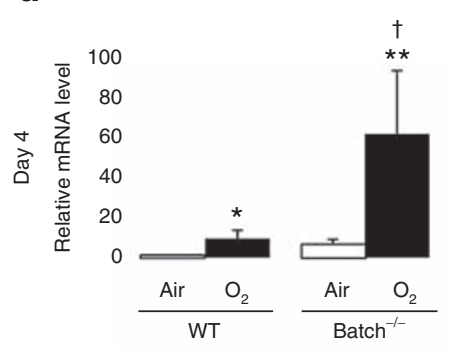

b

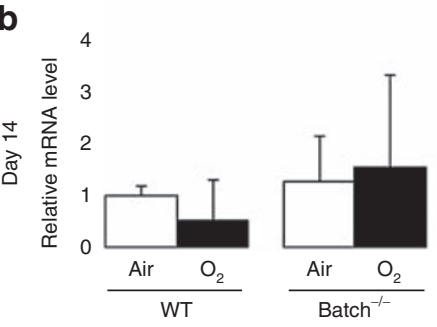

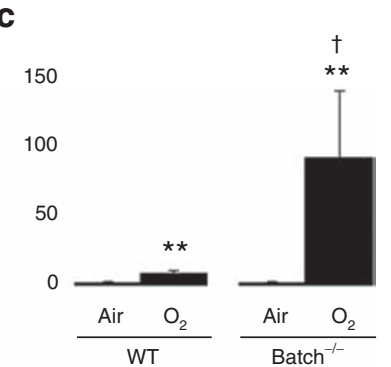

d

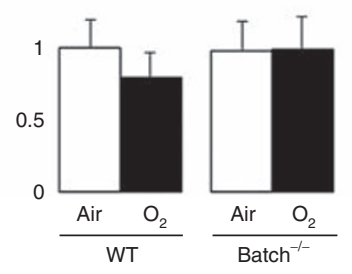

Figure 4. Gene expression levels of HO-1 and IL-6 in the lungs after neonatal hyperoxic exposure. Lung mRNA expression levels of $\mathrm{HO}-1(\mathbf{a}, \mathbf{b})$ and IL-6 (c, d) in Bach $1^{-1-}$ mice exposed to neonatal hyperoxia are enhanced compared with WT mice. quantitative RT-PCR was performed on day 4 and on day 14 ( $n=5$ in each group). Animals were exposed to air (open bars) or $\mathrm{O}_{2}$ (filled bars). Data are the mean $\pm \mathrm{SEM}$. Comparison between different groups was performed by one-way ANOVA followed by Tukey test. ${ }^{*} P$ $<0.05$ vs. Air, ${ }^{* *} P<0.01$ vs. Air, $+P<0.05$ vs. WT O 2. HO-1, heme oxygenase; IL-6, interleukin-6.

expression level was decreased to basal levels in WT and Bach $1^{-/}$mice (Figure 6).

\section{DISCUSSION}

In this study, Bach $1^{-/-}$mice were well-recovered from hyperoxic lung injury compared with WT mice. Bach $1^{-/-}$mice showed an enhanced recovery from delayed alveolar development, caused by neonatal hyperoxia, back to normal developmental levels. Furthermore, mRNA and/or protein levels of HO-1, IL-6, and MCP-1 in lungs from Bach $1^{-/-}$mice exposed to neonatal hyperoxia were significantly increased at $4 \mathrm{~d}$ of age compared with lungs from WT mice, suggesting that the recovery from hyperoxic lung injury observed in $\mathrm{Bach}^{-/-}$mice may be due to the antioxidant/anti-inflammatory activity of HO-1 and/ or transient proinflammatory cytokine overexpression that is strongly induced in $\mathrm{Bach}^{-/-}$mice.

Mice with a targeted HO-1 mutation show partial penetrance of embryonic lethality, growth retardation and a deficiency in iron metabolism (25-27). HO-1 null mutant mice were found to have mild alveolar simplification, disorganization, and reduced secondary crest formation. Another study also demonstrated that HO-1-null mice showed exaggerated hyperoxia-induced hypoalveolarization (28). These experiments demonstrate that HO- 1 is required for normal lung development. In contrast, HO-1 is well-known to be cytoprotective against redox stress and mitigate lung injury and alveolar simplification in hyperoxia-exposed neonatal mice. In a neonatal transgenic mouse model with constitutive lung-specific HO-1 overexpression, mice had attenuated pulmonary inflammation, arterial 
a

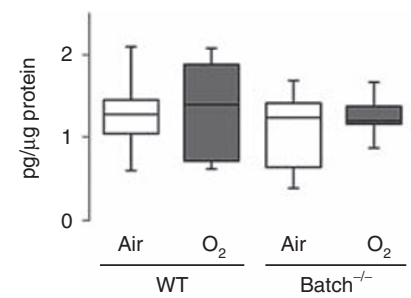

b

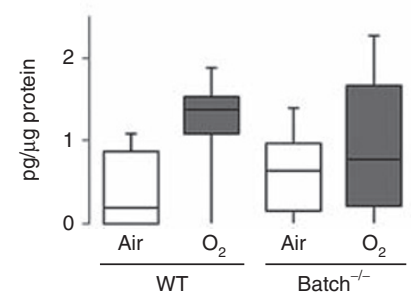

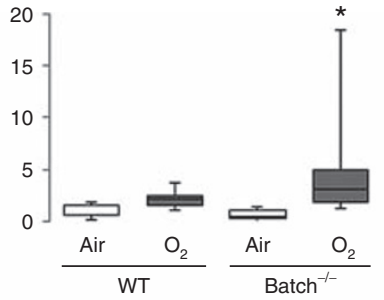

d

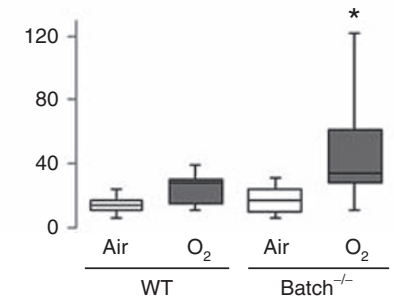

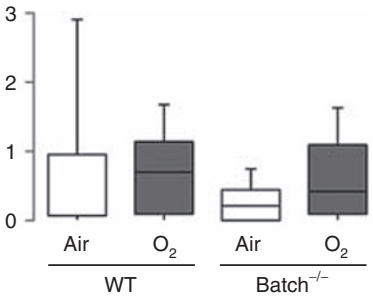

f

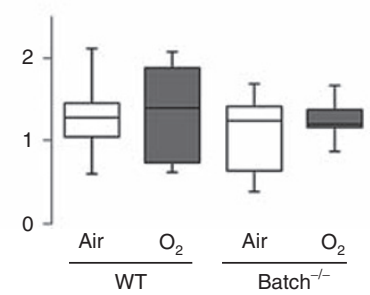

Figure 5. Protein levels of inflammatory cytokines in the lungs after neonatal hyperoxic exposure. Both IL-6 and MCP-1 levels in the Bach $1^{-/-}$mice lung were significantly higher than those of the WT mice lung following a 4-d hyperoxic exposure. Cytometric bead array was performed on day 4 ( $n=5$ in each group). Animals were exposed to air (open bars) or $\mathrm{O}_{2}$ (filled bars). Median protein levels of IFN-ã (a), IL-12 (b), IL-6 (c), MCP-1 (d), IL-10 (e), and TNF-á (f) in the lung homogenates. Box $=25^{\text {th }}$ and $75^{\text {th }}$ percentiles; bars $=$ min and max values. Comparison between different groups was performed by Kruskal-Wallis test followed by Mann-Whitney U-test. ${ }^{*}<0.05$ vs. Air. IFN, interferon; IL, interleukin; MCP-1, monocyte chemoattractant protein-1; TNF, tumor necrosis factor.

a

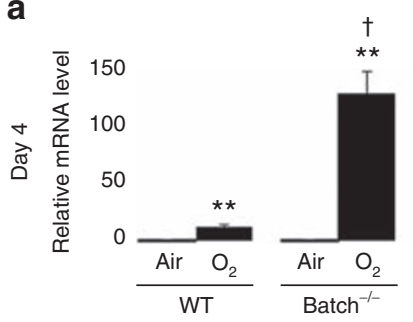

b

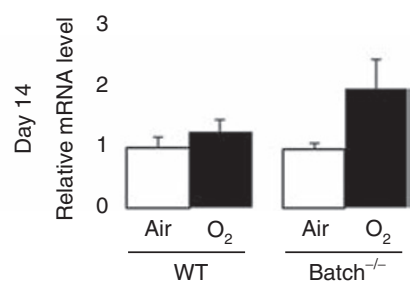

Figure 6. Gene expression levels of MCP-1 in the lungs after neonatal hyperoxic exposure. Lung mRNA expression levels of MCP-1 in Bach1 ${ }^{-1-}$ mice exposed to neonatal hyperoxia are enhanced compared with WT mice. quantitative RT-PCR was performed on day4 (a) and on day 14 (b). Animals were exposed to air (open bars) or $\mathrm{O}_{2}$ (filled bars). Data are the mean \pm SEM. Comparison between different groups was performed by one-way ANOVA followed by Tukey test. ${ }^{* *} P<0.01$ vs. Air, $\uparrow P<0.05$ vs. WT $\mathrm{O}_{2}$. MCP-1, monocyte chemoattractant protein-1.

remodeling, right ventricular hypertrophy, pulmonary edema, hemosiderosis, and a decrease in the number of blood vessels (29). While some degree of $\mathrm{HO}$ activity is cytoprotective, HO-1 expression levels that are too high were associated with significant oxygen cytotoxicity (30). Another study demonstrated that mice expressing high levels of $\mathrm{HO}-1$ had increased alveolar wall thickness with type II cell hyperproliferation and worsened pulmonary function after they recovered from hyperoxia (8). Overall, as regulators of cell proliferation and differentiation, moderate levels of HO-1 have a significant impact on both lung injury and lung repair processes in hyperoxia, suggesting that HO-1 expression levels in Bach1 ${ }^{-/-}$mice, both during and after neonatal hyperoxia, might be optimal to recover from hyperoxia-induced lung injury.

In this study, higher levels of IL-6 were induced in the lungs of $B a c h 1^{-1-}$ mice during neonatal hyperoxic exposure compared

with WT mice. Using transgenic adult mice that overexpress IL-6 in the lung, Ward et al. found that IL-6 markedly diminished hyperoxic lung injury and that this protection was associated with a marked decrease in hyperoxia-induced cell death and DNA fragmentation (31). Furthermore, Tanimoto et al. reported that the protective effects of IL- 6 against hyperoxia in $\mathrm{Bach}^{-/-}$mice may be mediated by the overexpression of IL-6 induced in the lungs after hyperoxic exposure (22). In contrast, in newborn mice, lung-targeted IL-6 overexpression was reported to cause significantly increased mortality, DNA injury, the induction of caspases, a cell death regulator, and the expression of angiogenic factors in hyperoxia (32).

In this study, despite IL-6 overexpression in the newborn lungs, $B a c h 1^{-/}$mice were well-recovered from lung injury induced by hyperoxic exposure. Two possibilities exist to explain this discrepancy. One is that HO-1 overexpression in the lungs of $\mathrm{Bach}^{-/-}$mice is significantly more effective to recover from hyperoxic exposure compared with IL-6 overexpression. The other possibility is that transient overexpression of IL-6 is beneficial to recover from hyperoxia-induced lung injury, whereas permanent or prolonged overexpression is harmful.

MCP-1 has chemotactic, homing, and activating effects on leukocytes. It is typically used as a marker of leukocyte chemotaxis. Previous reports have identified the production of MCP-1, and subsequent leukocyte infiltration, as pivotal events in the course of newborn hyperoxic lung injury (33). In this study, compared with WT mice, Bach $1^{-/-}$mice exposed to neonatal hyperoxia had significantly enhanced mRNA and protein levels of MCP-1 at $4 \mathrm{~d}$ of age and histologically better pulmonary outcomes during the recovery phase. Using $\mathrm{C}-\mathrm{C}$ chemokine receptor 2 (CCR2) deficient mice, Okuma et al. 
found that MCP-1/CCR2 signaling is protective against hyperoxia-induced lung injury, likely by suppressing the induction of inducible nitric oxide synthase and the consequent production of reactive oxygen species by activated alveolar macrophages (34). In this study, we found that the overexpression of MCP-1 in addition to HO-1 and IL-6 overexpression in the lungs of $\mathrm{Bach}^{-/-}$mice exposed to hyperoxia may play an important role in hyperoxic lung injury and the subsequent recovery process.

In conclusion, we found that $\mathrm{Bach} 1^{-/-}$neonatal mice are wellrecovered from hyperoxic lung injury during the recovery phase compared with WT mice. This recovery effect may be due to the antioxidant/anti-inflammatory activity of HO-1 and/or the transient overexpression of proinflammatory cytokines, such as IL-6 and MCP-1, which are strongly induced in $\mathrm{Bach}^{-/-}$mice.

\section{SUPPLEMENTARY MATERIAL}

Supplementary material is linked to the online version of the paper at http:// www.nature.com/pr

\section{STATEMENT OF FINANCIAL SUPPORT}

This work was supported by Saitama Medical Center Internal Research Grant for Young Physician Scientists (Grant no. 26F120) and Grants-in-Aid for Scientific Research (KAKENHI) from the Japan Society for the Promotion of Science (Grant no. 15K21334). The authors have no affiliations with or involvement in any organization or entity with any financial interest, or nonfinancial interest in the subject matter or materials discussed in this manuscript.

\section{REFERENCES}

1. Jobe AH, Kallapur SG. Long term consequences of oxygen therapy in the neonatal period. Semin Fetal Neonatal Med 2010;15:230-5.

2. Vogel ER, Britt RD Jr, Trinidad MC, et al. Perinatal oxygen in the developing lung. Can J Physiol Pharmacol 2015;93:119-27.

3. Maeda Y, Davé V, Whitsett JA. Transcriptional control of lung morphogenesis. Physiol Rev 2007;87:219-44.

4. Berger J, Bhandari V. Animal models of bronchopulmonary dysplasia. The term mouse models. Am J Physiol Lung Cell Mol Physiol 2014;307:L93647.

5. Warner BB, Stuart LA, Papes RA, Wispé JR. Functional and pathological effects of prolonged hyperoxia in neonatal mice. Am J Physiol 1998;275(1 Pt 1):L110-7.

6. Yee M, Chess PR, McGrath-Morrow SA, et al. Neonatal oxygen adversely affects lung function in adult mice without altering surfactant composition or activity. Am J Physiol Lung Cell Mol Physiol 2009;297:L641-9.

7. Yang G, Biswasa C, Lin QS, et al. Heme oxygenase-1 regulates postnatal lung repair after hyperoxia: role of $\beta$-catenin/hnRNPK signaling. Redox Biol 2013;1:234-43.

8. Namba F, Go H, Murphy JA, et al. Expression level and subcellular localization of heme oxygenase- 1 modulates its cytoprotective properties in response to lung injury: a mouse model. PLoS One 2014;9:e90936.

9. Bender AT, Ostenson CL, Wang EH, Beavo JA. Selective up-regulation of PDE1B2 upon monocyte-to-macrophage differentiation. Proc Natl Acad Sci USA 2005;102:497-502.

10. Fox S, Leitch AE, Duffin R, Haslett C, Rossi AG. Neutrophil apoptosis: relevance to the innate immune response and inflammatory disease. J Innate Immun 2010;2:216-27.

11. van Amerongen MJ, Harmsen MC, van Rooijen N, Petersen AH, van Luyn MJ. Macrophage depletion impairs wound healing and increases left ventricular remodeling after myocardial injury in mice. Am J Pathol 2007;170:818-29.

12. Leor J, Rozen L, Zuloff-Shani A, et al. Ex vivo activated human macrophages improve healing, remodeling, and function of the infarcted heart. Circulation 2006;114(1 Suppl):I94-100.
13. Jang HS, Kim J, Park YK, Park KM. Infiltrated macrophages contribute to recovery after ischemic injury but not to ischemic preconditioning in kidneys. Transplantation 2008;85:447-55.

14. Vandivier RW, Henson PM, Douglas IS. Burying the dead: the impact of failed apoptotic cell removal (efferocytosis) on chronic inflammatory lung disease. Chest 2006;129:1673-82.

15. Igarashi K, Hoshino H, Muto A, et al. Multivalent DNA binding complex generated by small Maf and Bach1 as a possible biochemical basis for betaglobin locus control region complex. J Biol Chem 1998;273:11783-90.

16. Sun J, Hoshino H, Takaku K, et al. Hemoprotein Bach1 regulates enhancer availability of heme oxygenase-1 gene. EMBO J 2002;21:5216-24.

17. Sun J, Brand M, Zenke Y, Tashiro S, Groudine M, Igarashi K. Heme regulates the dynamic exchange of Bach1 and NF-E2-related factors in the Maf transcription factor network. Proc Natl Acad Sci USA 2004;101:1461-6.

18. Ishikawa M, Numazawa S, Yoshida T. Redox regulation of the transcriptional repressor Bach1. Free Radic Biol Med 2005;38:1344-52.

19. Suzuki H, Tashiro S, Sun J, Doi H, Satomi S, Igarashi K. Cadmium induces nuclear export of Bach1, a transcriptional repressor of heme oxygenase-1 gene. J Biol Chem 2003;278:49246-53.

20. Omura S, Suzuki H, Toyofuku M, Ozono R, Kohno N, Igarashi K. Effects of genetic ablation of bach1 upon smooth muscle cell proliferation and atherosclerosis after cuff injury. Genes Cells 2005;10:277-85.

21. Yano Y, Ozono R, Oishi Y, et al. Genetic ablation of the transcription repressor Bach1 leads to myocardial protection against ischemia/reperfusion in mice. Genes Cells 2006;11:791-803.

22. Tanimoto T, Hattori N, Senoo T, et al. Genetic ablation of the Bach1 gene reduces hyperoxic lung injury in mice: role of IL-6. Free Radic Biol Med 2009;46:1119-26.

23. Hsia CC, Hyde DM, Ochs M, Weibel ER; ATS/ERS Joint Task Force on Quantitative Assessment of Lung Structure. An official research policy statement of the American Thoracic Society/European Respiratory Society: standards for quantitative assessment of lung structure. Am J Respir Crit Care Med 2010;181:394-418.

24. Yang G, Hinson MD, Bordner JE, et al. Silencing hyperoxia-induced C/ $\mathrm{EBP} \alpha$ in neonatal mice improves lung architecture via enhanced proliferation of alveolar epithelial cells. Am J Physiol Lung Cell Mol Physiol 2011;301:L187-96.

25. Poss KD, Tonegawa S. Reduced stress defense in heme oxygenase 1-deficient cells. Proc Natl Acad Sci USA 1997 30:10925-30.

26. Poss KD, Tonegawa S. Heme oxygenase 1 is required for mammalian iron reutilization. Proc Natl Acad Sci USA 1997;94:10919-24.

27. Duckers HJ, Boehm M, True AL, et al. Heme oxygenase-1 protects against vascular constriction and proliferation. Nat Med 2001;7:693-8.

28. Anyanwu AC, Bentley JK, Popova AP, et al. Suppression of inflammatory cell trafficking and alveolar simplification by the heme oxygenase-1 product carbon monoxide. Am J Physiol Lung Cell Mol Physiol 2014;306:L74963.

29. Fernandez-Gonzalez A, Alex Mitsialis S, Liu X, Kourembanas S. Vasculoprotective effects of heme oxygenase-1 in a murine model of hyperoxiainduced bronchopulmonary dysplasia. Am J Physiol Lung Cell Mol Physiol 2012;302:L775-84.

30. Suttner DM, Dennery PA. Reversal of HO-1 related cytoprotection with increased expression is due to reactive iron. FASEB J 1999;13:1800-9.

31. Ward NS, Waxman AB, Homer RJ, et al. Interleukin-6-induced protection in hyperoxic acute lung injury. Am J Respir Cell Mol Biol 2000;22:535-42.

32. Choo-Wing R, Nedrelow JH, Homer RJ, Elias JA, Bhandari V. Developmental differences in the responses of IL- 6 and IL-13 transgenic mice exposed to hyperoxia. Am J Physiol Lung Cell Mol Physiol 2007;293:L14250.

33. Vozzelli MA, Mason SN, Whorton MH, Auten RL Jr. Antimacrophage chemokine treatment prevents neutrophil and macrophage influx in hyperoxia-exposed newborn rat lung. Am J Physiol Lung Cell Mol Physiol 2004;286:L488-93.

34. Okuma T, Terasaki Y, Sakashita N, et al. MCP-1/CCR2 signaling pathway regulates hyperoxia-induced acute lung injury via nitric oxide production. Int J Exp Pathol 2006;87:475-83. 